\title{
Sérologie du lait : La lactoséro-agglutination rapide par centrifugation appliquée au diagnostic de la brucellose à partir des laits de grand mélange (1)
}

\author{
par \\ M. LE GUILLOUX \\ Directeur du Laboratoire Vétérinaire Départemental, \\ Maison de l'Agriculture, B.P. 1033 - 57000 Metz (France) \\ avec la collaboration de B. WATRIN et B. CAPS \\ techniciennes de laboratoire
}

Si le classique ring-test effectué sur des échantillons de lait de bidons de 10 à $20 \mathrm{l}$, correspondant à la traite de 4 à 8 vaches, est un test de grande valeur, on peut se demander quelle est sa sensibilité sur des échantillons de lait provenant de grands mélanges.

Les exploitants agricoles, encouragés par les industriels laitiers, s'équipent de plus en plus en tanks réfrigérés afin de réaliser une meilleure organisation du travail de part et d'autre.

Le diagnostic de la brucellose à partir de laits de grand mélange est donc d'actualité. Lors de la réunion annuelle des Directeurs des Laboratoires Vétérinaires Départementaux, en novembre 1972, nous eûmes l'occasion d'exposer notre expérience sur ce sujet, à savoir la pratique des réactions d'agglutination lente, d'agglutination rapide par centrifugation, de Coombs et de fixation du complément $(\mathrm{FC})$, réactions effectuées sur le lactosérum de laits bichromatés.

\section{DONNEES PREALABLES}

Chacun sait que l'on peut diagnostiquer la brucellose à partir du lait soit par l'épreuve de l'anneau avec le lait entier, soit par l'agglutination lente avec le lactosérum du lait écrémé puis emprésuré. Nous faisons mention ici d'autres techniques que nous avons étudiées

(1) Bulletin de la Société Vétérinaire Pratique, 28, rue des Petits-Hôtel - 75010 Paris, 1973, $\mathrm{n}^{\circ}$ 5, 221. 
et dont nous ignorons si elles ont déjà été utilisées. L'idée est de chercher à réaliser avec le lactosérum les mêmes réactions qu'avec le sérum sanguin. Nous nous sommes efforcés pour cela d'obtenir un lactosérum limpide. On peut y parvenir en emprésurant le lait normal à condition qu'il soit frais et écrémé. Mais, dans la pratique courante, il est préférable d'utiliser un conservateur, le bichromate de potassium. A la dilution de $1 / 2000$, nous constatons régulièrement qu'il conserve l'échantillon de lait plusieurs jours, qu'il n'altère ni les anticorps, ni les antigènes et qu'il ne possède pas de pouvoir anticomplémentaire. L'emprésurage du lait bichromaté est long, 1 à $2 \mathrm{~h}$ au bain-marie à $37^{\circ} \mathrm{C}$, mais le lactosérum obtenu est généralement limpide et l'on peut effectuer avec lui des réactions d'agglutination et de fixation du complément.

\section{LES REACTIONS UTILISEES SUR LES LAITS DE GRAND MELANGE}

Dans une première expérimentation réalisée en 1972 et portant sur des centaines d'échantillons de lait de tank, nous avons étudié comparativement les réactions mentionnées ci-après afin de rechercher qu'elles étaient les meilleures.

\section{$1^{\circ} \quad$ Les ring-tests}

Ils ont été effectués avec l'antigène Lacto-Bang de l'Institut Pasteur (suspension de brucelles tuées et colorées à l'hématoxyline). Les résultats ont été identiques en exécutant le test avec 2 et $3 \mathrm{ml}$ de lait ; avec $4 \mathrm{ml}$, on obtient 10 p. 100 de positivités supplémentaires.

\section{$2^{\circ}$ Les agglutinations avec le lactosérum}

L'agglutination lente en tubes et la réaction de Coombs à l'antiglobuline sont sans valeur par manque de sensibilité pour la première et par excès pour la seconde.

L'agglutination par centrifugation est la plus intéressante et sera décrite par la suite.

\section{$3^{\circ}$ La fixation du complément}

Elle a été effectuée avec $0,1 \mathrm{ml}$ de lactosérum selon notre technique d'hémolyse rapide [1]. Avec les laits de grand mélange, elle nous a déçus par manque de sensibilité.

Nous avons pu établir les corrélations suivantes sur les laits de grand mélange :

a) les résultats sont identiques entre l'agglutination par centrifugation effectuée avec $0,2 \mathrm{ml}$ de lactosérum et la fixation, mais 
ils sont inférieurs de $1 / 3$ aux résultats obtenus avec le ring-test sans positivités autres,

b) l'agglutination par centrifugation effectuée avec 0,5 $\mathrm{ml}$ de lactosérum donne des résultats identiques à ceux obtenus avec le ring-test,

c) l'agglutination par centrifugation est meilleure avec l'antigène coloré qu'avec l'antigène brucellique concentré,

d) l'agglutination par centrifugation effectuée avec $1 \mathrm{ml}$ ou $1,5 \mathrm{ml}$ de lactosérum possède une sensibilité supérieure à celle du ring-test habituel.

Après cette rapide orientation dans le choix des techniques, passons à la deuxième partie de notre expérimentation qui a eu pour but d'établir la valeur de notre technique comparativement à celle du ring-test.

\section{LA LACTOSERO-AGGLUTINATION RAPIDE PAR CENTRIFUGATION}

\section{Principe}

Il est identique au sérodiagnostic de la salmonellose par centrifugation. La réaction antigène-anticorps se déroule en deux temps : une phase d'union rapide mais inapparente ; une phase apparente, l'agglutination. La centrifugation accélère le processus, oblige les antigènes et les anticorps à se rencontrer et concentre les complexes dans le culot. Plus il y a de lactosérum, plus la réaction est sensible. C'est la réaction inverse de l'épreuve de l'anneau dans laquelle la crème fixe les complexes colorés.

\section{Caractères de l'agglutination}

L'agglutination brucellique est identique à l'agglutination somatique des salmonelles. Elle est granulaire et fine, d'où l'intérêt d'un antigène coloré ; elle est indissociable : l'agitation énergique du tube ne détruit pas les grains et permet de différencier les agglutinats vrais des particules de caillot parfois introduites.

\section{Technique}

Laisser au repos pendant quelques heures le flacon de lait de mélange frais ou bichromaté à $1 / 2000$ (bichromate de potassium).

1) Dans un tube à hémolyse, mettre une goutte d'extrait de

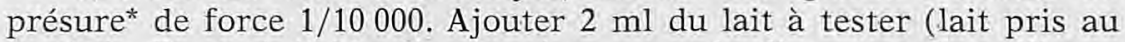
fond du flacon à l'aide d'une seringue munie d'une canule suffisam-

* Extrait de présure (Chr. Hausen), Etablissement Boll, 49, rue de la Bienfaisance - 94 Vincennes. 
ment longue; il faut éviter de prendre de la crème). Mélanger aussitôt. Mettre au bain-marie à $37^{\circ} \mathrm{C}$ pendant $2 \mathrm{~h}$.

2) Puis centrifuger à $3000 \mathrm{t}$ pendant $5 \mathrm{mn}$.

3) Recueillir le surnageant, environ $1 \mathrm{ml}$ à $1,5 \mathrm{ml}$, dans un autre tube à hémolyse.

4) Ajouter une goutte d'antigène coloré Lacto-Bang de l'Institut Pasteur dilué de moitié dans de l'eau physiologique.

5) Centrifuger à $3000 \mathrm{t}$ pendant $5 \mathrm{mn}$.

6) Après rejet du surnageant, ajouter à la seringue sur le culot $1 \mathrm{ml}$ d'eau distillée.

7) Agiter. Lecture :

- Réaction négative : liquide bleu, absence d'agglutinats.

- Réaction positive : liquide éclairci, présence d'agglutinats.

En laissant les tubes au repos, les agglutinats apparaissent nettement au fond des tubes positifs.

\section{LES RESULTATS OBTENUS}

Ils concernent les laits provenant de mélanges supérieurs à 1001 dont la répartition approximative a été la suivante :

1001 à $2001 \ldots \ldots \ldots 30$

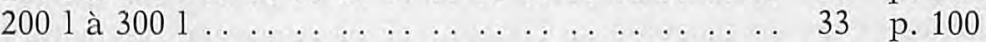

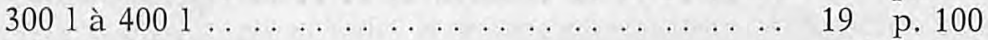

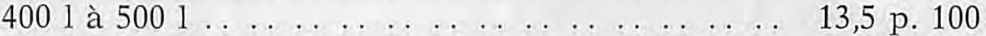

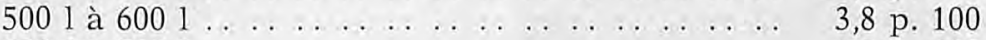

6001 à $7001 \ldots \ldots \ldots \ldots$. . . . . . . . . . . . . . . . . . . 100

En anticipant, disons tout de suite que les positivités supplémentaires révélées par la centrifugation se situent dans toutes les catégories.

Le tableau 1 indique les résultats comparatifs obtenus entre le ring-test effectué avec $2 \mathrm{ml}$ et la lactoséro-agglutination rapide par centrifugation.

TABLEAU 1

\begin{tabular}{c|c|c}
\hline $\begin{array}{c}\text { Nombre de tanks } \\
\text { testés : } 1012\end{array}$ & $\begin{array}{c}\text { Lactoséro-agglutinations rapides } \\
\text { Positives 279 }\end{array}$ & Négatives 783 \\
$\begin{array}{c}\text { Ring-tests positifs } \\
225 \\
20,2 \text { p. } 100\end{array}$ & 223 & 2 \\
Ring-tests négatifs & & \\
787 & 56 & 731 \\
\hline
\end{tabular}




\section{Constatations}

1) Les positivités des ring-tests sont incluses dans celles des agglutinations, sauf 2 .

2) Aux 225 positivités des ring-tests s'ajoutent 56 positivités révélées par la centrifugation, soit 25 p. 100 en plus.

Il reste à vérifier la fidélité de ces constatations.

\section{Le contrôle sérologique}

1) Ces 56 positivités nouvelies correspondent-elles à des étables brucelliques?

Il y a eu 21 étables contrôlées par prises de sang sur les bovins âgés de plus de 2 ans, étables classées ci-dessous selon leur pourcentage d'animaux reconnus positifs.

\begin{tabular}{|c|c|}
\hline $\begin{array}{l}\text { Nombre d'animaux } \\
\text { âgés de plus de } 2 \text { ans }\end{array}$ & $\begin{array}{c}\text { Nombre d'animaux positifs } \\
\geqslant 72 \text { UI ou FC positive }\end{array}$ \\
\hline $\begin{array}{l}\text { Exploitation : } \mathrm{n}^{\circ} \quad 1=44 \\
\mathrm{n}^{\circ} \quad 2=28 \\
\mathrm{n}^{\circ} \quad 3=23 \\
\mathrm{n}^{\circ} \quad 4=30 \\
\mathrm{n}^{\circ} \quad 5=30 \\
\mathrm{n}^{\circ} \quad 6=45 \\
\mathrm{n}^{\circ} \quad 7=17 \\
\mathrm{n}^{\circ} \quad 8=84 \\
\mathrm{n}^{\circ} \quad 9=47 \\
\mathrm{n}^{\circ} 10=29 \\
\mathrm{n}^{\circ} 11=61 \\
\mathrm{n}^{\circ} 12=37 \\
\mathrm{n}^{\circ} 13=19 \\
\mathrm{n}^{\circ} 14=58 \\
\mathrm{n}^{\circ} 15=41 \\
\mathrm{n}^{\circ} 16=21 \\
\mathrm{n}^{\circ} 17=42 \\
\mathrm{n}^{\circ} 18=71 \\
\mathrm{n}^{\circ} 19=54 \\
\mathrm{n}^{\circ} 20=13 \\
\mathrm{n}^{\circ} 21=23\end{array}$ & $\begin{aligned} 15 \\
9 \\
7 \\
7 \\
6 \\
8 \\
3 \\
10 \\
5 \\
3 \\
4 \\
2 \\
1 \\
3 \\
2 \\
1 \\
2 \\
3 \\
1 \\
\text { Animaux suspects } \\
\text { 2 (60 UI ; FC nég.) } \\
1 \text { (30 UI ; FC nég.) }\end{aligned}$ \\
\hline
\end{tabular}

On constate que la sérologie confirme les positivités supplémentaires révélées par l'agglutination rapide. On s'explique mal l'échec du ring test dans les 10 premières exploitations. La technique par centrifugation possède apparemment une grande sensibilité puisqu'elle détecte un taux faible d'infection (cas des 9 dernières exploitations). 
2) L'agglutination rapide a été défaillante dans deux cas révélés par le ring-test :

- Exploitation $n^{\circ} 22=55$ vaches .. . . 16 animaux positifs,

- Exploitation no $23=39$ vaches .. . . . 2 animaux positifs.

Elle connaît donc apparemment des échecs :

- En ce qui concerne les laits provenant de mélanges inférieurs à 1001 , on retrouve à peu près les mêmes différences significatives entre les résultats obtenus par les deux méthodes.

- Cette étude ne présente pas de conclusion définitive. Il existe encore trop de rapports à déterminer. Nous n'avons eu que peu souvent l'occasion de contrôler les animaux de troupeaux reconnus négatifs par cette technique d'agglutination. D'autre part, si celle-ci présente une nette supériorité sur le ring-test habituel exécuté avec $2 \mathrm{ml}$ de lait, nous connaissons insuffisamment sa valeur comparativement au ring-test exécuté avec un volume double de lait qui comble une grande partie du déficit enregistré avec le ring-test habituel.

Dans ce travail, nous avons essayé de montrer les nombreuses possibilités de diagnostic sérologique de la brucellose avec le lait. La technique d'agglutination que nous avons décrite, et dont nous avons montré la valeur, trouve sa place dans le chapitre de la sérologie du lait.

\section{Bibliographie}

[1] Le Guilloux (M.) (1972). - Une nouvelle réaction d'hémolyse rapide appliquée au diagnostic de la Brucellose, de la Salmonellose et de la maladie de Johne chez les bovins. Revue Méd. Vét., 123, 1589. 